\title{
Expression and localization of MrgprD in mouse intestinal tract
}

\author{
Chenxing Zhou ${ }^{1} \cdot$ Jia Li $^{1} \cdot$ Lin Liu $^{1} \cdot$ Zongxiang Tang ${ }^{2} \cdot$ Fengyi Wan ${ }^{3,4,5} \cdot$ Lei Lan $^{1}$
}

Received: 2 October 2018 / Accepted: 9 March 2019 / Published online: 27 March 2019

(C) The Author(s) 2019

\begin{abstract}
MrgprD, a Mas-related G protein-coupled receptor, is initially identified in sensory neurons of mouse dorsal root ganglia (DRG) and has been suggested to participate in somatosensation. However, MrgprD has recently been found to be expressed outside the nervous system such as in aortic endothelia cells and neutrophils. In this study, we used immunohistochemistry to detect the expression and localization of MrgprD in mouse intestinal tract. The immunoreactivity (IR) of MrgprD was found in the smooth muscle layers of small intestine, colon and rectum. In addition, MrgprD IR was colocalized with F4/80-positive macrophages and CD3-positive T lymphocytes resident in the lamina propria of intestinal mucosa. MrgprD was also found to be expressed in primary peritoneal macrophages and splenic T lymphocytes. Furthermore, the presence of MrgprD mRNA and its protein was detected in murine macrophage-like RAW 264.7 and human T lymphocyte Jurkat cell lines. Our study shows, for the first time, the expression and localization of MrgprD in the intestinal tract and in macrophages and T lymphocytes, indicating the potential roles of MrgprD in intestinal mobility and immunity.
\end{abstract}

Keywords MrgprD $\cdot$ Intestinal tract $\cdot$ Smooth muscle cells $\cdot$ Macrophages $\cdot$ T lymphocytes

\section{Introduction}

MrgprD, also termed as MrgD or TGR7, is one member of the Mas-related $\mathrm{G}$ protein-coupled receptor (Mrgpr) family, which is highly expressed in small-diameter sensory neurons of dorsal root ganglia (DRG) (Dong et al.
2001). MrgprD is found in the majority of unmyelinated nociceptive neurons that are labeled by isolectin B4 and express ATP-gated ion channel P2X3 in DRG neurons (Zylka et al. 2003, 2005). MrgprD-expressing neurons exclusively innervate the outer layers of mammalian skin and are necessary for behavioral hypersensitivity to

Electronic supplementary material The online version of this article (https://doi.org/10.1007/s00441-019-03017-7) contains supplementary material, which is available to authorized users.

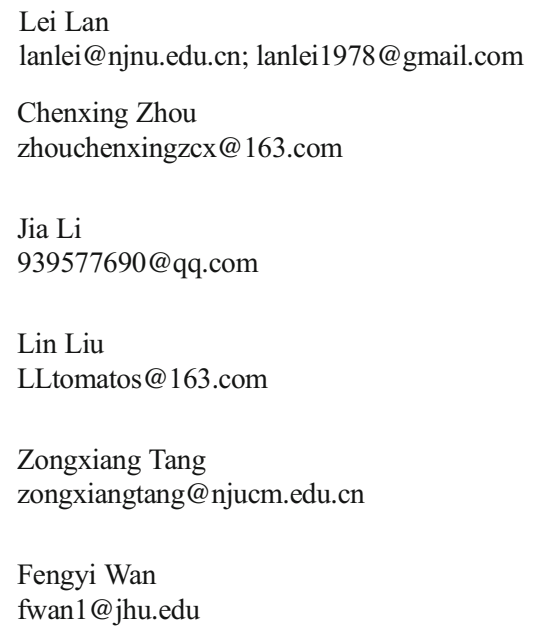

1 Jiangsu Province Key Laboratory for Molecular and Medical Biotechnology, College of Life Sciences, Nanjing Normal University, Nanjing 210023, Jiangsu, People's Republic of China

2 Key Laboratory of Chinese Medicine for Prevention and Treatment of Neurological Diseases, School of Medicine and Life Sciences, Nanjing University of Chinese Medicine, Nanjing 210023, Jiangsu, People's Republic of China

3 Department of Biochemistry and Molecular Biology, Bloomberg School of Public Health, Johns Hopkins University, Baltimore, MD 21205, USA

4 Department of Molecular Microbiology and Immunology, Bloomberg School of Public Health, Johns Hopkins University, Baltimore, MD 21205, USA

5 Department of Oncology and Sidney Kimmel Comprehensive Cancer Center, Johns Hopkins University, Baltimore, MD 21205 , USA 
noxious stimuli (Cavanaugh et al. 2009). MrgprD is activated by $\beta$-alanine (Shinohara et al. 2004) and has been found to inhibit KCNQ/M-type potassium channels and increase excitability of sensory neurons (Crozier et al. 2007). Genetic deletion of MrgprD significantly decreased the sensitivity of cutaneous nociceptors to mechanical and thermal stimuli (Rau et al. 2009). Specifically, a decreased firing rate was observed in cells lacking Mrgprd in response to lower mechanical forces and noxious heat. Thresholds of activation were remarkably lower in response to cold and higher in response to hot stimuli. The application of $\beta$-alanine significantly reduced the rheobase and increased the firing rate in the neurons of $\mathrm{Mrgprd}^{+/-}$heterozygous mice but not in Mrgprd $^{-1}$ mice. Thus, MrgprD was originally considered as a modulator of cell excitability in response to mechanical and thermal stimuli. Recently, Liu et al. reported that MrgprD-expressing C-fiber mechanosensitive neurons mediate $\beta$-alanine-evoked histamine-independent itch (Liu et al. 2012). Clearly, MrgprD is involved in somatosensation and/or modulation.

The expression and function of MrgprD outside the nerve system has been reported. MrgprD is expressed in aortic endothelia cells and has been identified as a receptor for alamandine, a new component of renin-angiotensin system (RAS) on the regulation of cardiovascular homeostasis (Habiyakare et al. 2014; Lautner et al. 2013). It has been suggested that alamandine, the peptide identified as being generated by catalysis of angiotensin A (Ang A) via angiotensin-converting enzyme 2 (ACE2) or directly by aspartate decarboxylation from Ang-(1-7), produced endothelial-dependent vasodilation in rat and mice aortic rings through MrgprD (Etelvino et al. 2014). Additionally, MrgprD has been found expressed in mouse primary neutrophils (Da Silva et al. 2017). The treatment of alamandine reduced inflammation in carotid atherosclerotic plaques probably by inhibition of neutrophil degranulation, the first sign of presence of MrgprD in immune cells. However, the detailed expression pattern and the physiological roles of MrgprD in inflammation remain largely elusive.

Recently, two reports from the same group documented the enteric neuronal expressions of subtypes of the Mrgpr family in the normal and inflamed mouse ileums (Avula et al. 2011, 2013). The authors showed that the inflammatory condition causes the changes in the distributions of Mrgpr subtypes, i.e., MrgprA, MrgprB, MrgprC, MrgprE, MrgprF, MrgprG and $\mathrm{MrgprH}$, in murine ileum, suggesting that these receptors may be involved in the mediation of neuroimmune communication during intestinal inflammation. However, the information about intestinal expression and localization of MrgprD is lacking to date. Thus, in this study, we sought to determine whether MrgprD is expressed in the intestinal tract and we found an intense expression of MrgrpD in the muscular wall and in mucosal macrophages and lymphocytes.

\section{Materials and methods}

\section{Animals}

Animal experiments and care were performed in strict compliance with the guidelines outlined within the Guide to Animal Use and Care from the Nanjing Normal University. $\mathrm{C} 57 \mathrm{BL} / 6$ mice were housed in groups of five per cage in our animal center, with free access to food and water under a 12-h light-dark cycle.

\section{Isolation of peritoneal macrophages and splenic T lymphocytes}

The C57BL/ 6 mice at $8-10$ weeks were injected with $1-2 \mathrm{ml}$ of $5 \%$ starch broth $(0.25 \mathrm{~g}$ beef extract, $1 \mathrm{~g}$ peptone and $0.5 \mathrm{~g}$ sodium chloride in $100 \mathrm{ml}$ distilled water) 3 days before the extraction of peritoneal macrophages. The mice were sacrificed by cervical dislocation under anesthesia and sterilized by soaking in $75 \%$ ethanol for $3 \mathrm{~min}$. The $5 \mathrm{ml}$ of RPMI 1640 medium was injected into the abdomen of the mouse and massaged for $3 \mathrm{~min}$. Then, a small incision was cut under the abdomen of the mouse and the peritoneal fluid was sucked out with a pipette tip. Cell suspension was centrifuged at $1000 \mathrm{rpm}$ for $5 \mathrm{~min}$ and washed thrice with PBS solution. Then, the cells were cultured with RPMI 1640 containing $10 \%$ fetal bovine serum. Unadherent cells were removed after incubation for $3 \mathrm{~h}$ in the incubator.

The mice were sacrificed by cervical dislocation under anesthesia and sterilized by soaking in 75\% ethanol for $3 \mathrm{~min}$; then, the spleen was excised under a sterile environment. The spleen tissue was ground and the lymphocyte separation solution (Multisciences) was added. The homogenate was centrifuged at $400 \times g$ for $20 \mathrm{~min}$ at room temperature. After centrifugation, the homogenate was divided into four layers from top to bottom and the second white layer is the lymphocyte layer. Then, the CD3-positive $\mathrm{T}$ lymphocytes were insolated from the second layer using MagCellect Mouse $\mathrm{CD}^{+} \mathrm{T}$ Cell Isolation Kit (R\&D, MAGM201) following the manufacturer's instructions and finally cultured in RPMI 1640 containing $10 \%$ FBS.

\section{Immunohistochemistry and confocal microscopy}

The intestinal tracts and aorta from 8 to 10 -week-old mice were fixed in $4 \%$ paraformaldehyde in $1 \times$ PBS at room temperature for $2.5 \mathrm{~h}$, cryoprotected in $30 \%$ sucrose in $1 \times$ PBS overnight and embedded into OCT. Tissues were cut into sections at a thickness of $10 \mu \mathrm{m}$, air dried and stored at $-80{ }^{\circ} \mathrm{C}$. For immunostanining of MrgprD, CD3 and $\mathrm{F} 4 / 80$, sections were incubated in blocking solution (containing 10\% donkey serum (Solarbio, Beijing, China), $0.3 \%$ Triton $\mathrm{X}-100$ in $\mathrm{PBS}$ ) for $1 \mathrm{~h}$ at room temperature 
and then incubated with rabbit anti-MrgprD (1:100, Abcam, ab155099) and/or rat anti-CD3 (1:200, Abcam, ab11089) or rat anti-F4/80 (1:200, Abcam, ab6640), respectively, at $4{ }^{\circ} \mathrm{C}$ overnight. Next, to detect the MrgprD expression, the sections were washed and incubated with Alexa Fluor 555-conjugated donkey anti-rabbit secondary antibody (1:200, Invitrogen, A31572) at $37{ }^{\circ} \mathrm{C}$ for $1 \mathrm{~h}$. To detect the colocalization of MrgprD with F4/80 or CD3, the sections were washed and incubated with Alexa Fluor 555-conjugated donkey anti-rabbit (1:200) and Alexa Fluor 488-conjugated donkey anti-rat IgG secondary antibody (1:200, Invitrogen, A21208) at $37{ }^{\circ} \mathrm{C}$ for $1 \mathrm{~h}$. For negative controls, the primary antibodies were omitted and replaced by blocking solution followed by incubation with corresponding secondary antibodies. Lastly, the cell nuclei were counterstained with DAPI for $5 \mathrm{~min}$ at room temperature. The pictures were captured using an Axio Zoom V16 microscope (ZEISS).

The primary or cultured cells were fixed in $4 \%$ paraformaldehyde at room temperature for $20 \mathrm{~min}$, permeabilized with $0.5 \%$ Triton X-100 and were blocked for $40 \mathrm{~min}$ in PBS containing $5 \%$ bovine serum albumin. The fixed cells were then incubated with rabbit anti-MrgprD (1:500, Abcam, ab155099) and/or rat anti-CD3 (1:200, Abcam, ab11089), rat anti-F4/80 (1:200, Abcam, ab6640) and mouse anti-SM-MHC11 (1:200, smooth muscle myosin heavy chain 11 , Abcam, ab683), respectively, in blocking buffer at $4{ }^{\circ} \mathrm{C}$ overnight. After washing, cells were incubated with the secondary antibodies of Alexa Fluor 647-conjugated donkey anti-rabbit (to detect MrgprD, 1:500, Abcam, ab150075) and/or Alexa Fluor 488conjugated goat anti-rat (to detect F4/80 or CD3, 1:500, Abcam, ab 150157) or Alexa Fluor 488-conjugated donkey anti-mouse IgG secondary antibody (to detect SM-MHC11, 1:500, Abcam, ab150105) at $37^{\circ} \mathrm{C}$ for $1 \mathrm{~h}$ in the dark, followed by three times washing ( $0.1 \%$ Triton X-100 in PBS). For negative controls, the primary antibodies were omitted and replaced by blocking solution followed by incubation with corresponding secondary antibodies. Lastly, the cell nuclei were counterstained with DAPI. Slides were mounted and examined under a Nikon A1 confocal laser microscope system (Tokyo, Japan).

\section{Cell culture and protein extraction}

The murine macrophage-like RAW 264.7 (ATCC TIB71) and human $\mathrm{T}$ lymphocyte Jurkat cell lines (ATCC TIB-152) were cultured in DMEM (Wisent Corporation) and RIPM 1640 (Wisent Corporation) supplement with $10 \%$ fetal bovine serum (Wisent Corporation), $100 \mathrm{U} / \mathrm{ml}$ penicillin and $100 \mu \mathrm{g} / \mathrm{ml}$ streptomycin (Wisent Corporation) at $37{ }^{\circ} \mathrm{C}$ in an atmosphere of $5 \% \mathrm{CO}_{2}$. The cells were maintained by passaging every 2 days.
Freshly isolated DRGs, livers, or cultured cells were homogenized with cell lysis buffer (Beyotime, P0013J) containing proteinase inhibitor cocktail (Abcam, ab201119) and incubated on ice for $30 \mathrm{~min}$. Cell lysates were centrifuged $(12,500 \mathrm{rpm})$ at $4{ }^{\circ} \mathrm{C}$ for $15 \mathrm{~min}$. The extracted proteins were used in subsequent experiments.

\section{RNA isolation and reverse transcription PCR}

Total RNA was extracted from freshly isolated DRGs, liver, thoracic aorta or cell lines using TRIzol reagent (Invitrogen) and the contaminated DNA was digested using DNase I (Roche). Complementary DNA (cDNA) was produced with PrimeScript RT reagent kit (TaKaRa). The sequences of the mouse Mrgprd primers were as follows: forward, 5'-TTTTCAGTGACATT CCTCGCC-3', and reverse, 5'-GCACATAGACACAG AAGGGAGA-3'. The sequences of the mouse GAPDH primers were as follows: forward, 5'-TGAA GGTCGGTGTGAACGGATTT-3' and reverse, 5'-TGGT TCACACCCATCACAACAT-3'. All samples for each gene were run at least in triplicate.

\section{Western blot analysis}

The protein extracted from murine intestinal tissues or different cell lines were mixed with Laemmli $2 \times$ loading buffer. Proteins were separated by SDS-PAGE and then transferred onto a nitrocellulose membrane (Whatman, GE Healthcare, NJ, USA). The membrane was blocked with $5 \%$ non-fat dry milk in TBS-Tween-20 $(0.1 \%, v / v)$ for $2 \mathrm{~h}$ at room temperature. After briefly washing with cold TBS, the membrane was probed with the indicated primary antibody (the dilution used according to the manufacturer's instructions) overnight at $4{ }^{\circ} \mathrm{C}$, followed by three times washing. The antibody-antigen complexes were visualized by the LI-COR Odyssey Infrared Imaging System according to the manufacturer's instruction using IRDye800 fluorophore-conjugated antibody (LI-COR Biosciences, Lincoln, NE, USA).

\section{Nuclear-cytoplasmic separation}

Raw 264.7 and Jurkat cells ( $10^{6}$ per dish) were plated in 6-cm dishes. The cells were washed with PBS for three times and lysed with lysis buffer. The lysates were incubated on ice for $20 \mathrm{~min}$ and centrifuged at $3000 \times \mathrm{g}$ for $10 \mathrm{~min}$ at $4{ }^{\circ} \mathrm{C}$. The supernatants (cytoplasmic extracts) were collected and pellets were washed and resuspended with RIPA buffer. After incubation on ice for $30 \mathrm{~min}$, the nuclear extracts were collected by centrifugation at $12,000 \times \mathrm{g}$ for $20 \mathrm{~min}$. The nuclear and cytoplasmic fractions were resolved on $12 \%$ SDS-PAGE and subjected to Western blotting. 


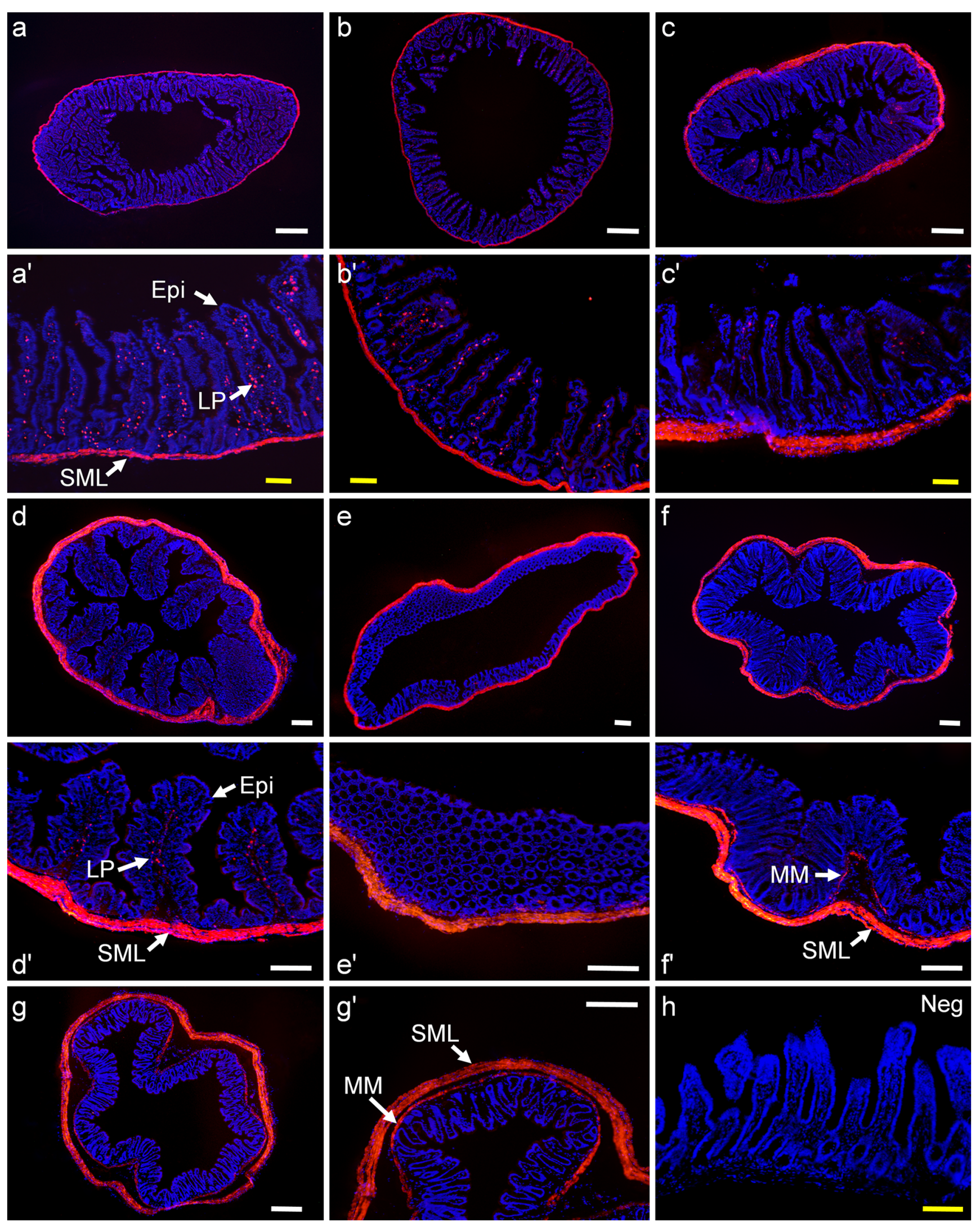


Fig. 1 MrgprD expression pattern in murine intestinal tract determined by immunohistochemistry. (a-g) Immunostaining of MrgprD (red) in the different segments of intestinal tract from anterior to posterior, i.e., duodenum (a), jejunum (b), ileum (c), proximal colon (d), middle colon (e), distal colon ( $\mathrm{f}$ ) and rectum $(\mathrm{g})$. ( $\left.\mathrm{a}^{\prime}-\mathrm{g}^{\prime}\right)$ The higher magnification images corresponding to $(\mathrm{a}-\mathrm{g})$ are shown, respectively. MrgprD IR was found in the smooth muscle layers (SML), muscularis mucosae (MM) and the lamina propria (LP) of the intestinal tract but not in the epithelium (Epi). (h) The negative control showing the absence of MrgprD IR in the ileum. The nuclei were counterstained with DAPI. Scale bars, $500 \mu \mathrm{m}$ (in white) and $100 \mu \mathrm{m}$ (in yellow)

\section{Results}

\section{MrgprD expression in small intestine, colon and rectum}

The antibody against MrgprD was used to detect the expression pattern of MrgprD protein in murine intestinal tract by immunohistochemistry. The intestinal tissues were dissected and divided into different segments, i.e., duodenum (Fig. 1a), jejunum (Fig. 1b), ileum (Fig. 1c), proximal colon (Fig. 1d), middle colon (Fig. 1e), distal colon (Fig. 1f) and rectum (Fig. 1g). MrgprD IR was detected in the smooth muscle layers throughout the intestinal tract (Fig. 1a-g) and in the lamina propria of the intestinal mucosa of small and large intestines (Fig. 1a'-f'). The MrgprD antibody was specific, as MrgprD IR was absent in negative control sections omitting primary antibody (Fig. 1h and ESM Fig. 1), while MrgprD IR was clearly located in a subset of DRG neurons (ESM Fig. 2).

It has been illustrated that MrgprD is expressed in smooth muscle cells of abdominal aorta from rabbits (Habiyakare et al. 2014). We further confirmed that MrgprD was expressed in the smooth muscle layers of murine thoracic aorta (Fig. 2a-a"). Using confocal investigation, we showed that MrgprD was colocalized with SM-MHC11, a specific marker for smooth muscle cells (Xu et al. 2017), in the primary smooth muscle cells isolated from murine colon (Fig. $2 \mathrm{~b}-\mathrm{b}^{\prime \prime}$ ). The negative controls showed no staining observed in isolated smooth muscle cells when the primary antibody was omitted (ESM Fig. 3a, b).

\section{Localization of MrgprD in mucosal macrophages and lymphocytes}

Additionally, we observed that MrgprD immunostaining exhibited a punctate distribution in the lamina propria of the intestinal mucosa (e.g., Fig. 1a', d'). It has been well documented that the intestinal lamina propria is rich in macrophages and lymphocytes, which are essential to mucosal immunity (Egan et al. 2011; Hirotani et al. 2005; Klein et al. 2017). To identify the cell types of MrgrpD-positive cells located in the intestinal lamina propria, we used antibodies against $\mathrm{F} 4 / 80$ and CD3 to label macrophages and $\mathrm{T}$ lymphocytes in the lamina propria, respectively. Using double immunohistochemical staining, we showed that MrgprD immunoreactive cells were largely colocalized with immunostainings of F4/80 (Fig. 3a-f) and CD3 (Fig. 3g-1) in the lamina propria, respectively, indicating that $\mathrm{MrgprD}$ is expressed in the resident macrophages and $\mathrm{T}$ lymphocytes in intestinal mucosa. The negative controls demonstrated the specificities of antibodies used since no staining was observed on the sections of ileum and colon when the primary antibody was omitted (ESM Fig. 3c-f).

\section{MrgprD expression in the peritoneal macrophages and the splenic $\mathrm{T}$ lymphocytes}

To investigate whether MrgprD is expressed in peripheral macrophages and $\mathrm{T}$ lymphocytes, the peritoneal macrophage
Fig. 2 MrgprD is expressed in intestinal smooth muscle cells. $\left(\mathrm{a}-\mathrm{a}^{\prime \prime}\right)$ Immunostaining of MrgprD (red) in murine thoracic aorta. Bright-field image of cryostate section of aorta (a), the staining of anti-MrgprD in aorta $\left(a^{\prime}\right)$ and the higher magnification view of a' showing distribution of MrgprD in smooth muscle layers of aorta $\left(a^{\prime \prime}\right)$. (b-b") Double immunostaining of mouse SMMHC11 (green, b) and MrgprD (red, $b^{\prime}$ ) in the primary smooth muscle cells isolated from murine colon. The nuclei were counterstained with DAPI. Scale bars $100 \mu \mathrm{m}$
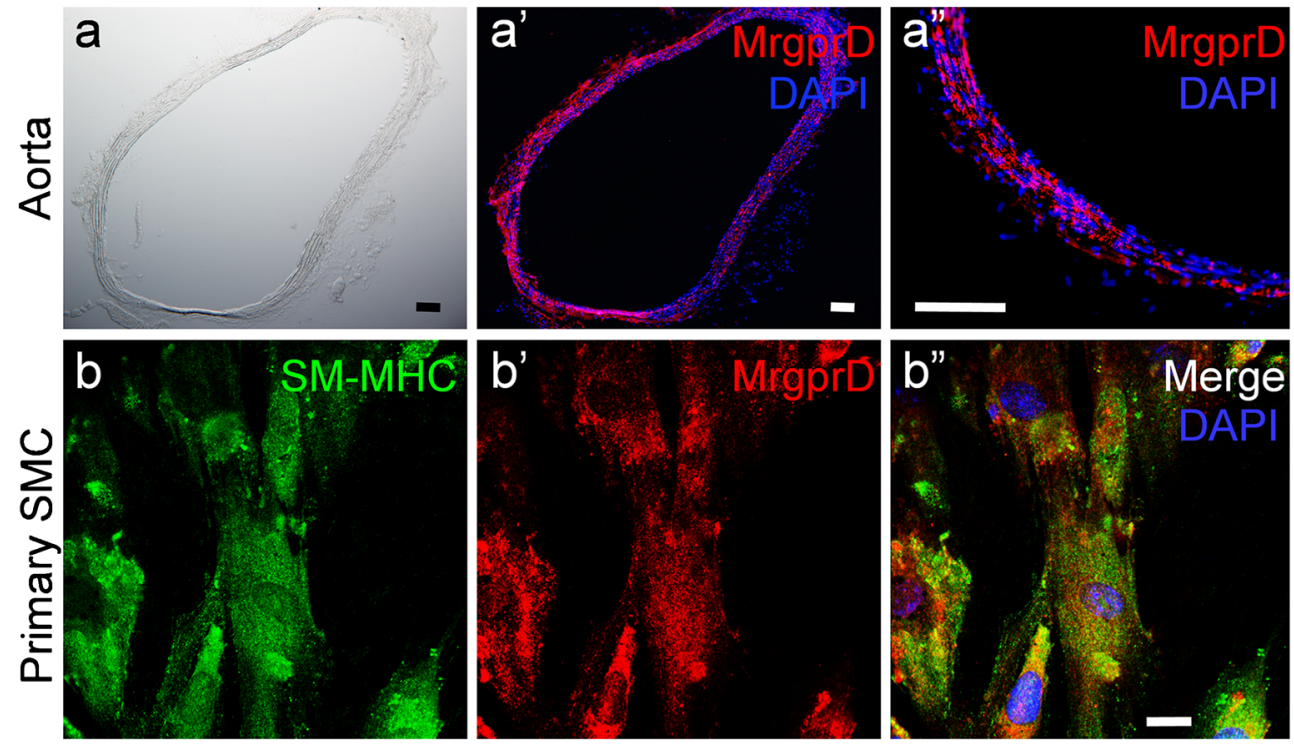

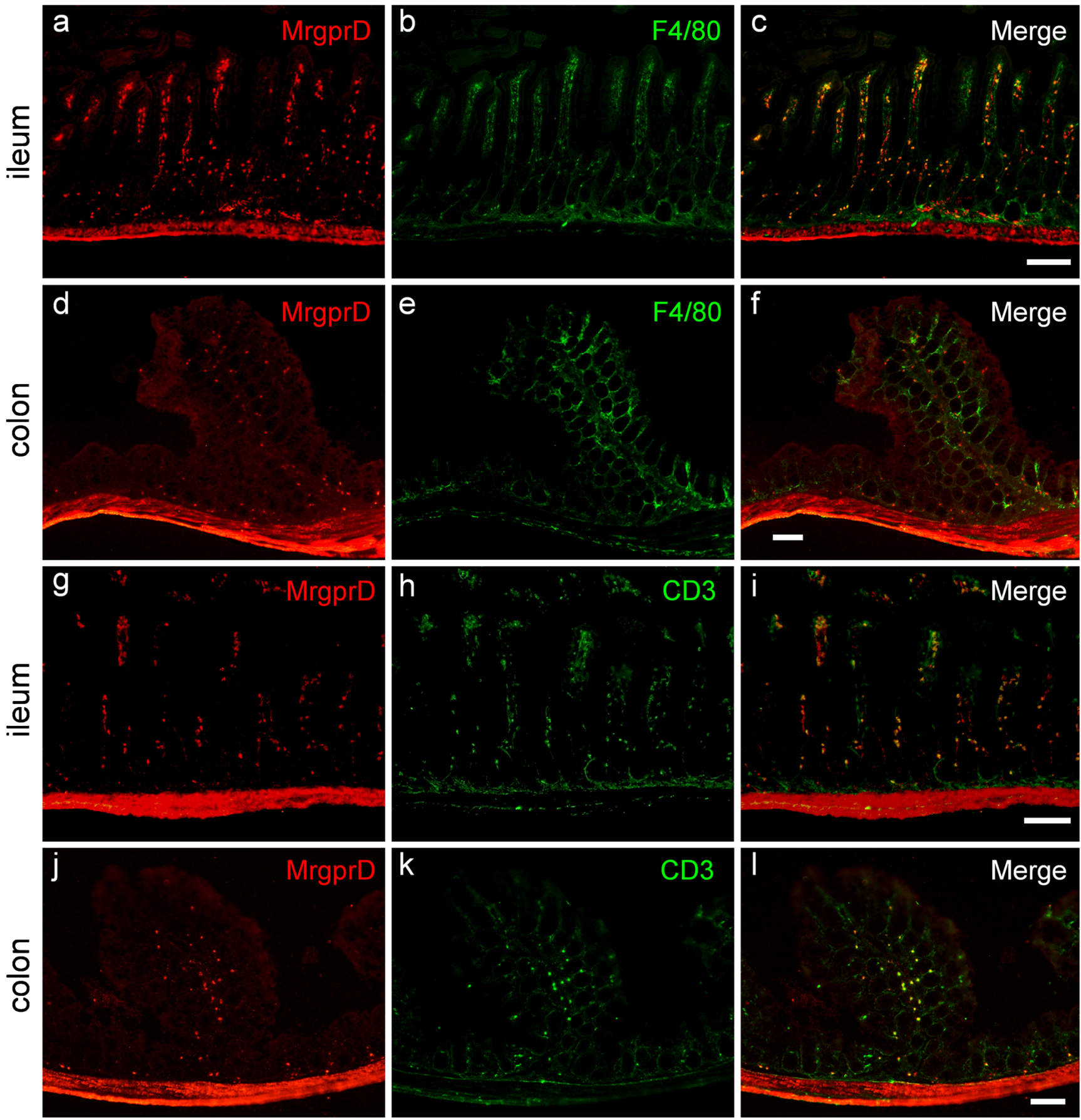

Fig. 3 MrgprD is expressed in the resident macrophages and $\mathrm{T}$ lymphocytes in lamina propria of intestinal mucosa. a-f Double immunostaining using anti-MrgprD and anti-F4/80 showed the expression of MrgprD (red) in the F4/80-positive macrophages (green) in the

lamina propria of ileum $(\mathbf{a}-\mathbf{c})$ and colon $(\mathbf{d}-\mathbf{f})$, respectively. $\mathbf{g}-\mathbf{l}$ Double immunostaining using anti-MrgprD and anti-CD3 showed the expression of MrgprD (red) in the CD3-positive T lymphocytes (green) in the lamina propria of ileum $(\mathbf{g}-\mathbf{i})$ and colon $(\mathbf{j}-\mathbf{l})$, respectively. Scale bars $100 \mu \mathrm{m}$

cells were isolated from abdomen of mice and the CD3-positive $\mathrm{T}$ lymphocytes were purified from mouse spleen as described in the "Materials and methods" section. Using RT-PCR, we found that Mrgprd mRNA was present in the peritoneal macrophages and the $\mathrm{CD} 3$-positive $\mathrm{T}$ lymphocytes as well as in DRG, aorta and in the primary smooth muscle cells but not in liver (Fig. 4a). In addition, using confocal investigation, we showed that MrgprD protein was expressed in the primary peritoneal macrophages positive for F4/80 expression (Fig. $\left.4 \mathrm{~b}-\mathrm{b}^{\prime \prime \prime}\right)$ and the splenic $\mathrm{T}$ lymphocytes positive for CD3 expression (Fig. $4 \mathrm{c}-\mathrm{c}^{\prime \prime \prime}$ ). 


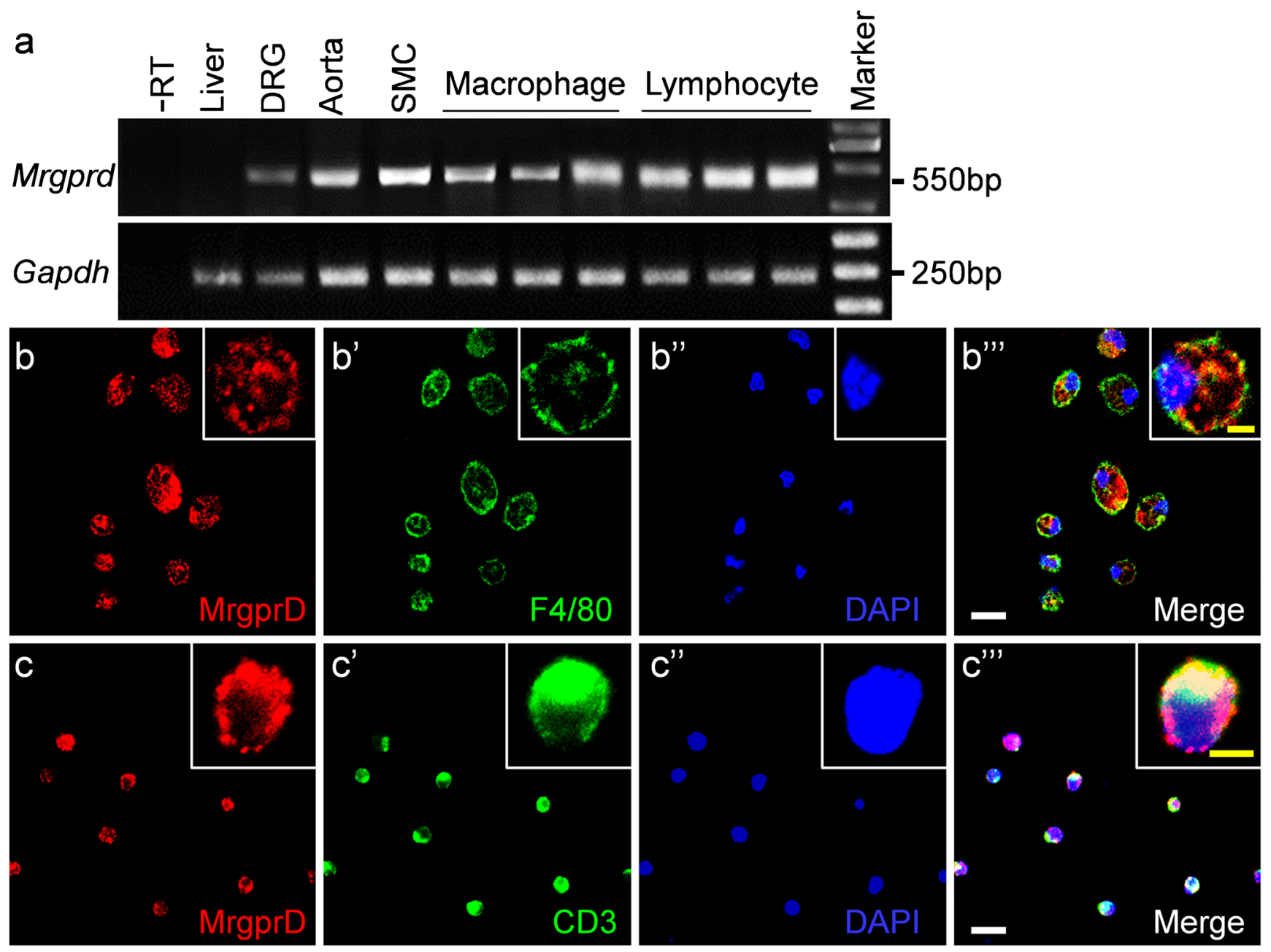

Fig. 4 Expressions of MrgprD in peritoneal macrophages and splenic T lymphocytes. a RT-PCR analyses to detect the expressions of Mrgprd in liver, DRG, aorta, primary smooth muscle cells (SMC), primary peritoneal macrophages (in triplicates) and splenic $\mathrm{T}$ lymphocytes (in triplicates). - RT was the negative control omitting the template. The liver was used as the control negative for Mrgprd expression and DRG and aorta were used as the positive controls. $\left(b-b^{\prime \prime \prime}\right)$ Double

\section{Presence of MrgprD in RAW 264.7 and Jurkat cell lines}

To further examine the expression of MrgprD in immune cells, the murine macrophage-like RAW 264.7 and human $\mathrm{T}$ lymphocyte Jurkat cell lines were used. Both the results of RT-PCR (Fig. 5a) and Western blot analyses (Fig. 5b) revealed the expression of MrgprD in RAW 264.7 and Jurkat cells. The presence of MrgprD protein in these cells was further confirmed by confocal microscopy (Fig. $5 \mathrm{c}-\mathrm{c}^{\prime \prime}, \mathrm{d}-\mathrm{d}^{\prime \prime}$ ). We also noticed that a small amount of MrgprD immunostaining was present in the nuclei of Jurkat cells (Fig. 5d"). We thus separately isolated the cytosolic and nuclear fractions from RAW 264.7 and Jurkat cells, respectively and detected whether MrgprD protein is expressed in the cell nucleus using Western immunohistochemical stainings showing the expression of MrgprD protein (red, $b$ and $b^{\prime \prime \prime}$ ) in F4/80-positive peritoneal macrophages (green, $b^{\prime}$ and $\left.\mathrm{b}^{\prime \prime \prime}\right)$. (c- $\left.\mathrm{c}^{\prime \prime \prime}\right)$ Double immunohistochemical stainings showing the expression of MrgprD protein (red, c and $\mathrm{c}^{\prime \prime \prime}$ ) in CD3-positive T lymphocytes (green, $\mathrm{c}^{\prime}$ and $\mathrm{c}^{\prime \prime \prime}$ ). The nuclei were counterstained with DAPI. Higher magnification views of a representative cell are shown as insets in each panel. Scale bars: $20 \mu \mathrm{m}$ and $5 \mu \mathrm{m}$ in inserts

blotting. The results showed that MrgprD is expressed in the cytoplasm but not nuclei of Raw 264.7 and Jurkat cells (Fig. 5e).

\section{Discussion}

MrgprD, a member of the Mas-related G protein-coupled receptor (Mrgpr) family, was initially found to be expressed in a subset of nociceptors in mouse DRG in 2001 (Dong et al. 2001). In recent years, growing evidence suggest that MrgprD may participate in the modulation and/or sensation of pain (Tiwari et al. 2016) and itch (Meixiong and Dong 2017). The present study, for the first time, examines the expression and localization of MrgprD in murine intestinal tract 
Fig. 5 The expressions of MrgprD in murine macrophagelike RAW 264.7 and human T lymphocyte Jurkat cell lines. (a, b) The expressions of MrgprD in RAW 264.7 and Jurkat cells were determined by RT-PCR (a) and Western blotting (b). The tissue lysate of liver was used as the negative control and the lysate of DRGs was the positive control. $\left(\mathrm{c}-\mathrm{c}^{\prime \prime}\right.$ and $\left.\mathrm{d}-\mathrm{d}^{\prime \prime}\right)$ Confocal images showed the presence of MrgprD protein (red) in RAW 264.7 (c-c") and Jurkat cells ( $\left.\mathrm{d}-\mathrm{d}^{\prime \prime}\right)$. Scale bars $20 \mu \mathrm{m}$. (e) The cytoplasmic/ nuclear fractions were separately extracted from Raw 264.7 and Jurkat cells (in triplicates) and analyzed for the expressions of MrgprD, Lamin B and GAPDH by Western blotting. The expressions of MrgprD and GAPDH but not Lamin B, in cytoplasmic fractions were detected (upper panels). By contract, MrgprD was not expressed in the nuclear fractions of Raw 264.7 and Jurkat cells. The purity of nuclear fraction was determined by the expression of Lamin B and the absence of GAPDH (lower panels)

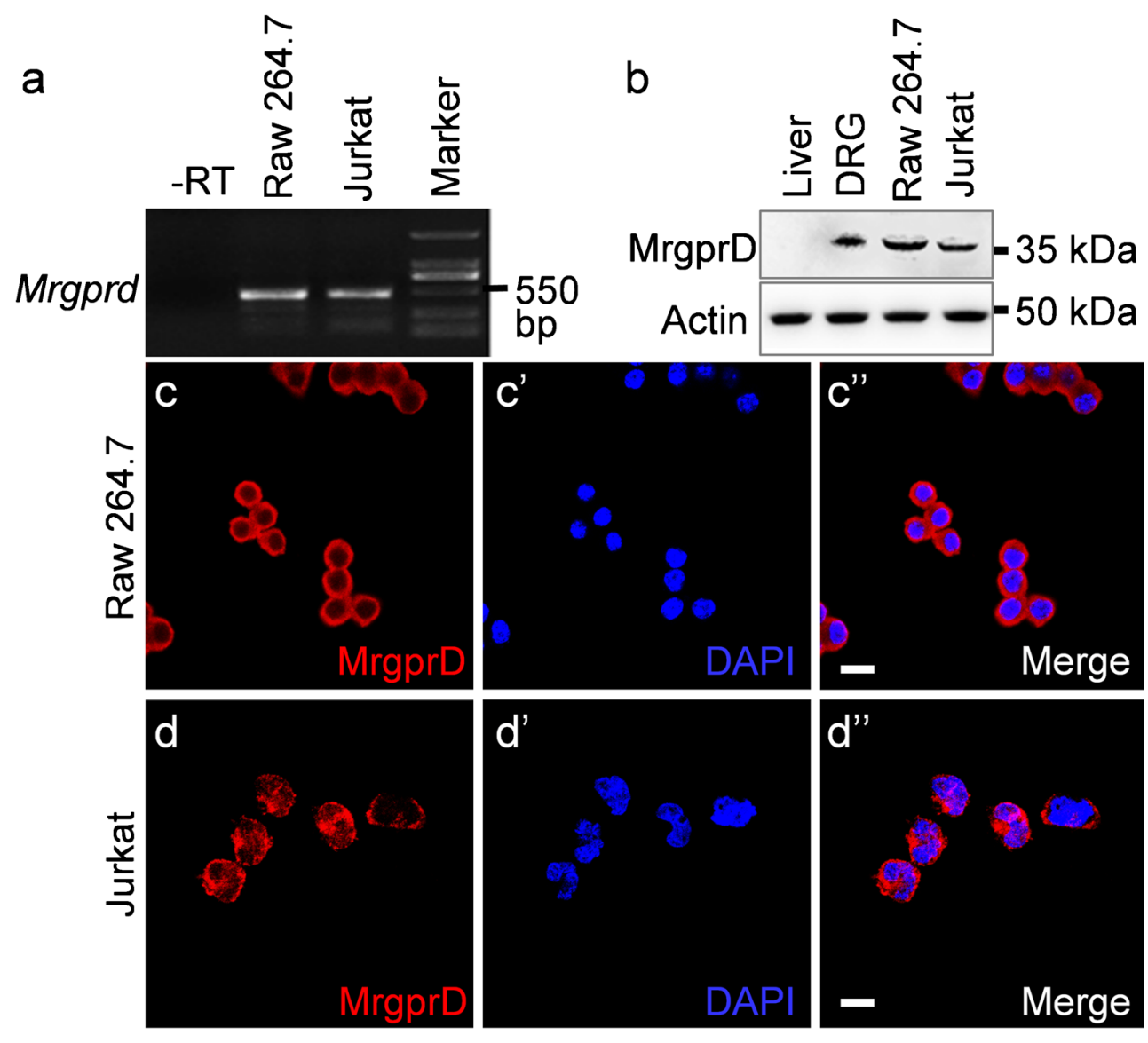

e

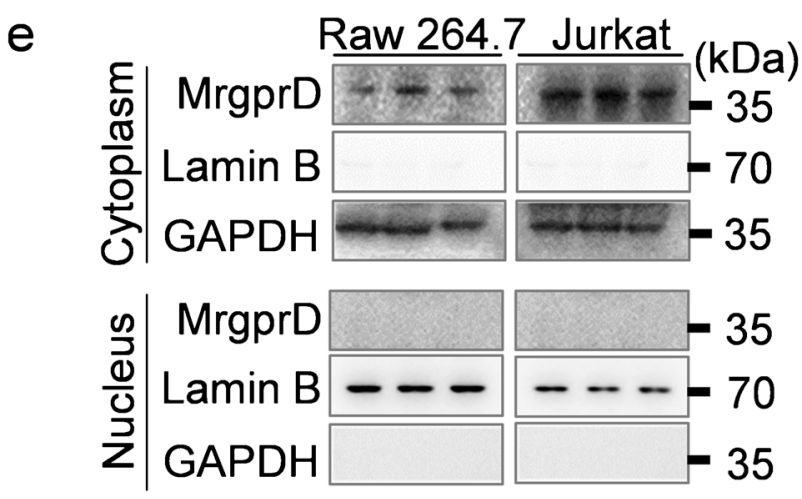

and shows the presence of MrgprD in smooth muscle cells, intestinal mucosal and peripheral immune cells, specifically, macrophages and $\mathrm{T}$ lymphocytes.

In the intestinal tract, a strong immunoreactive signal of MrgprD was observed in the smooth muscle layers. We further showed that MrgprD was also expressed in the smooth muscle layers of thoracic aorta. This observation is partially in agreement with the previous report of MrgprD expression only observed in the smooth muscle cells within atherosclerotic plaques in diseased aorta (Habiyakare et al. 2014) but we found a strong immunostaining of MrgprD in the smooth muscle layers of normal aorta. A possible explanation for this discrepancy is that, while the abovementioned study used paraffin-embedded tissues and the different MrgprD antibody (sc-138439; Santa Cruz, USA), we used cryostat sections probed with MrgprD antibody from Abcam. Additionally, using primary smooth muscle cells isolated from colon, we confirmed the presence of MrgprD in smooth muscle cells. In DRG neurons, activation of MrgprD by its ligand $\beta$-alanine caused calcium influx and excitation of MrgprD-positive neurons (Liu et al. 2012; Zhuo et al. 2014). Therefore, it is intriguing to investigate whether MrgprD is involved in the mobility and contractility of intestinal smooth muscle cells.

We showed, for the first time, the localization of MrgprD in the intestinal lamina propria. The punctate distribution of MrgprD immunostainings was readily observed in the lamina 
propria of small intestine and proximal colon. Interestingly, we identified that the MrgprD IRs were localized in the F4/80positive macrophages and CD3-positive $\mathrm{T}$ lymphocytes in the lamina propria of both ileum and colon. It has been demonstrated that Runx1, a Runt-domain transcription factor, plays a pivotal role in determining nociceptive sensory neuron phenotypes (Chen et al. 2006; Yoshikawa et al. 2007). The conditional knockout of Runxl resulted in loss of Mrgprd expression in DRG neurons (Abdel Samad et al. 2010) and, accordingly, Runx1 expression was persistent in most of MrgprD-positive neurons (Qi et al. 2017). Of note, the Runx1 transcription factor was first found in leukemic cells and is one of the key factors that drives various aspects of $\mathrm{T}$ cell differentiation (Wong et al. 2011). In addition, Runx 1 regulates the cell proliferation and survival of macrophages (Himes et al. 2005). We therefore determined the expression of Runx 1 in the intestinal tract. As expected, a similar punctate distribution of Runx 1 immunostaining was readily observed in the intestinal lamina propria; however, Runx1 expression did not exhibit in smooth muscle layers (data not shown). We speculate that Runx1 expression may be involved in the presence of MrgprD expression in mucosal resident immune cells in murine intestinal tract.

Since the Mrgprd gene was originally discovered based on its expression in DRG neurons, we also tried to determine whether MrgprD is expressed in enteric neurons. We used anti-NeuN antibody (ab104224, Abcam) to label enteric neurons on the sections of murine intestinal tract. Unfortunately, this antibody did not work. In addition, we cannot distinguish the location of MrgprD in plexus myentericus because of strong immunostaining of anti-MrgprD in smooth muscle layers throughout the intestinal tract. Therefore, it is required to further analyze MrgprD-positive cells located in the intestinal lamina propria and muscle layers using approaches such as flow cytometry to provide a comprehensive profile of diverse cell types with MrgprD expression.

Moreover, we showed that MrgprD was also expressed the peripheral peritoneal macrophage cells and splenic T lymphocytes. One previous study reported the mRNA expression of Mrgprd in mouse primary neutrophils isolated from ApoE knockout mice (Da Silva et al. 2017). We moreover showed, for the first time, both mRNA and protein expressions of MrgprD in peripheral macrophage cells and $\mathrm{T}$ lymphocytes. The expression of MrgprD in these immune cells implies a potential role of MrgprD in inflammatory response not only in systemic inflammation but also in intestinal immunity. The presence of MrgprD in the murine macrophage-like RAW264.7 and human T lymphocyte Jurkat cell lines will facilitate the investigation of a functional link between MrgprD and inflammatory response in future studies.

Collectively, our study revealed an expression pattern of MrgprD in murine intestinal tract, laying the foundation for further study on potential roles of MrgprD in intestinal mobility and immunity.
Authors' contributions L.Lan and $\mathrm{CZ}$ conceived and designed the experiments; L.Lan, CZ, JL and L.Liu performed the experiments; L.Lan, FW and ZT analyzed the data; CZ and L.Lan wrote the paper.

Funding information This work was supported by grants from the National Natural Science Foundation of China (No. 31500628 and No. 31870131), Natural Science Foundation of Jiangsu Province (No. BK20161555), National Institutes of Health (GM111682) to FW, the Priority Academic Program Development of Jiangsu Higher Education Institutions (PAPD) to Department of Life Science, Nanjing Normal University and sponsored by "Qing Lan Project" to L.Lan from Nanjing Normal University.

Open Access This article is distributed under the terms of the Creative Commons Attribution 4.0 International License (http:// creativecommons.org/licenses/by/4.0/), which permits unrestricted use, distribution and reproduction in any medium, provided you give appropriate credit to the original author(s) and the source, provide a link to the Creative Commons license and indicate if changes were made.

\section{References}

Abdel Samad O, Liu Y, Yang FC, Kramer I, Arber S, Ma Q (2010) Characterization of two Runx1-dependent nociceptor differentiation programs necessary for inflammatory versus neuropathic pain. Mol Pain 6:45

Avula LR, Buckinx R, Alpaerts K, Costagliola A, Adriaensen D, Van Nassauw L, Timmermans JP (2011) The effect of inflammation on the expression and distribution of the MAS-related gene receptors $\mathrm{MrgE}$ and $\mathrm{MrgF}$ in the murine ileum. Histochem Cell Biol 136: 569-585

Avula LR, Buckinx R, Favoreel H, Cox E, Adriaensen D, Van Nassauw L, Timmermans JP (2013) Expression and distribution patterns of Mas-related gene receptor subtypes A-H in the mouse intestine: inflammation-induced changes. Histochem Cell Biol 139:639-658

Cavanaugh DJ, Lee H, Lo L, Shields SD, Zylka MJ, Basbaum AI, Anderson DJ (2009) Distinct subsets of unmyelinated primary sensory fibers mediate behavioral responses to noxious thermal and mechanical stimuli. Proc Natl Acad Sci U S A 106:9075-9080

Chen CL, Broom DC, Liu Y, de Nooij JC, Li Z, Cen C, Samad OA, Jessell TM, Woolf CJ, Ma Q (2006) Runx1 determines nociceptive sensory neuron phenotype and is required for thermal and neuropathic pain. Neuron 49:365-377

Crozier RA, Ajit SK, Kaftan EJ, Pausch MH (2007) MrgD activation inhibits KCNQ/M-currents and contributes to enhanced neuronal excitability. J Neurosci 27:4492-4496

Da Silva AR, Lenglet S, Carbone F, Burger F, Roth A, Liberale L, Bonaventura A, Dallegri F, Stergiopulos N, Santos RA, Mach F, Fraga-Silva RA, Montecucco F (2017) Alamandine abrogates neutrophil degranulation in atherosclerotic mice. Eur J Clin Investig 47: $117-128$

Dong X, Han S, Zylka MJ, Simon MI, Anderson DJ (2001) A diverse family of GPCRs expressed in specific subsets of nociceptive sensory neurons. Cell 106:619-632

Egan C, Maurer K, Cohen S, Mack M, Simpson K, Denkers E (2011) Synergy between intraepithelial lymphocytes and lamina propria $\mathrm{T}$ cells drives inflammation during infection. J Immunol 186

Etelvino GM, Peluso AA, Santos RA (2014) New components of the renin-angiotensin system: alamandine and the MAS-related G protein-coupled receptor D. Curr Hypertens Rep 16:433 
Habiyakare B, Alsaadon H, Mathai ML, Hayes A, Zulli A (2014) Reduction of angiotensin A and alamandine vasoactivity in the rabbit model of atherogenesis: differential effects of alamandine and Ang(1-7). Int J Exp Pathol 95:290-295

Himes SR, Cronau S, Mulford C, Hume DA (2005) The Runx1 transcription factor controls CSF-1-dependent and -independent growth and survival of macrophages. Oncogene 24:5278-5286

Hirotani T, Lee PY, Kuwata H, Yamamoto M, Matsumoto M, Kawase I, Akira S, Takeda K (2005) The nuclear IkappaB protein IkappaBNS selectively inhibits lipopolysaccharide-induced IL-6 production in macrophages of the colonic lamina propria. J Immunol 174:3650-3657

Klein JR, Williams A, Vigneswaran N, Montufar-Solis D (2017) Lamina propria lymphocytes in sanroque mice produce proinflammatory cytokines that drive chronic small intestinal inflammation. $\mathrm{J}$ Immunol 198

Lautner RQ, Villela DC, Fraga-Silva RA, Silva N, Verano-Braga T, CostaFraga F, Jankowski J, Jankowski V, Sousa F, Alzamora A, Soares E, Barbosa C, Kjeldsen F, Oliveira A, Braga J, Savergnini S, Maia G, Peluso AB, Passos-Silva D, Ferreira A, Alves F, Martins A, Raizada M, Paula R, Motta-Santos D, Klempin F, Pimenta A, Alenina N, Sinisterra R, Bader M, Campagnole-Santos MJ, Santos RA (2013) Discovery and characterization of alamandine: a novel component of the renin-angiotensin system. Circ Res 112:1104-1111

Liu Q, Sikand P, Ma C, Tang Z, Han L, Li Z, Sun S, LaMotte RH, Dong X (2012) Mechanisms of itch evoked by beta-alanine. J Neurosci 32: $14532-14537$

Meixiong J, Dong X (2017) Mas-related G protein-coupled receptors and the biology of itch sensation. Annu Rev Genet 51:103-121

Qi L, Huang C, Wu X, Tao Y, Yan J, Shi T, Cao C, Han L, Qiu M, Ma Q, Liu Z, Liu Y (2017) Hierarchical specification of pruriceptors by runt-domain transcription factor Runx1. J Neurosci 37:5549-5561

Rau KK, McIlwrath SL, Wang H, Lawson JJ, Jankowski MP, Zylka MJ, Anderson DJ, Koerber HR (2009) Mrgprd enhances excitability in specific populations of cutaneous murine polymodal nociceptors. J Neurosci 29:8612-8619

Shinohara T, Harada M, Ogi K, Maruyama M, Fujii R, Tanaka H, Fukusumi S, Komatsu H, Hosoya M, Noguchi Y, Watanabe T, Moriya T, Itoh Y, Hinuma S (2004) Identification of a G proteincoupled receptor specifically responsive to beta-alanine. J Biol Chem 279:23559-23564

Tiwari V, Tiwari V, He S, Zhang T, Raja SN, Dong X, Guan Y (2016) Mas-related G protein-coupled receptors offer potential new targets for pain therapy. Adv Exp Med Biol 904:87-103

Wong WF, Kohu K, Chiba T, Sato T, Satake M (2011) Interplay of transcription factors in T-cell differentiation and function: the role of Runx. Immunology 132:157-164

Xu JG, Zhu SY, Heng BC, Dissanayaka WL, Zhang CF (2017) TGFbeta1-induced differentiation of SHED into functional smooth muscle cells. Stem Cell Res Ther 8:10

Yoshikawa M, Senzaki K, Yokomizo T, Takahashi S, Ozaki S, Shiga T (2007) Runx 1 selectively regulates cell fate specification and axonal projections of dorsal root ganglion neurons. Dev Biol 303:663-674

Zhuo RG, Ma XY, Zhou PL, Liu XY, Zhang K, Wei XL, Yan HT, Xu JP, Zheng JQ (2014) Mas-related G protein-coupled receptor D is coupled to endogenous calcium-activated chloride channel in Xenopus oocytes. J Physiol Biochem 70:185-191

Zylka MJ, Dong X, Southwell AL, Anderson DJ (2003) Atypical expansion in mice of the sensory neuron-specific Mrg G protein-coupled receptor family. Proc Natl Acad Sci U S A 100:10043-10048

Zylka MJ, Rice FL, Anderson DJ (2005) Topographically distinct epidermal nociceptive circuits revealed by axonal tracers targeted to Mrgprd. Neuron 45:17-25

Publisher's note Springer Nature remains neutral with regard to jurisdictional claims in published maps and institutional affiliations. 